\title{
Security Model for Distributed Object Framework and its Applicability to CORBA
}

\author{
Vijay Varadharajan \\ Professor of Computing \\ Distributed System and Network Security Research Group, \\ University of Western Sydney - Nepean, \\ PO Box 10, Kingswood, NSW 2747, Australia \\ Tel: $+6147360192, \quad$ Fax: +6147360800 \\ email: vijay@st.nepean.uws.edu.au
}

\section{Thomas Hardjono}

Centre for Computer Security Research, University of Wollongong, Wollongong, NSW 2522, Australia

Tel: +6142 214327, Fax: +6142 214329

email: thomas@cs.uow.edu.au

\begin{abstract}
Distributed object systems represent the next generation of distributed systems, integrating the recent development in object-oriented technology with the traditional area of distributed computing. In this paper we discuss the security issues related to distributed object systems in the context of the Common Object Request Broker Architecture (CORBA) framework. We extend an existing secure distributed object model, and present these extensions and details of the model within the CORBA framework. We describe the security services and identify the components of the model that provide these services. Authentication of objects, secure creation and deletion of objects, access control, and delegation between objects are presented. The security model is applicable to distributed object-oriented applications in general, and in particular to implementations of distributed object systems conforming to the CORBA standard.
\end{abstract}

\section{Keywords}

Distributed Objects, Information Security, Access Control, CORBA. 


\section{INTRODUCTION}

Distributed computing systems entered a new era when the concept of distributed object systems was introduced. This progress is only natural and inevitable, as object concepts have been in vogue in a number of traditional computing research areas during most of the last decade. A number of research projects in distributed object systems and object management systems have been in progress for the past few years (eg. Kim, 1990). In the more specific area of security, much of the research work have been based more on object-oriented database systems (eg. Fernandez, Gudes and Song (1989), Keefe and Tsai (1989), Lunt (1989), Thuraisingham (1989)). Some research on the security of distributed objects in the general sense have been reported by Varadharajan and Black (1991) and Varadharajan (1992).

A number of factors make the object concept attractive from the point of view of systems development and maintenance. The computing industry itself has recognized these advantages and the object-oriented technology is increasingly being adopted by companies producing hardware and software alike. An important standard for object-oriented systems and applications is the Object Management Architecture (OMA) put forward by the Object Management Group (OMG), which is a consortium of over 400 software vendor and object technology user companies. Part of that standard which focusses on application interoperability is the Common Object Request Broker Architecture (CORBA).

Within the context of the OMA/CORBA standard the issue of security has received considerable attention. Recently, a White Paper on security (OMG, 1994) was introduced by the OMG, focusing on a number requirements for security in the OMA. Some of the requirements summarized by OMG (1994) are as follows: User authentication, Security in communications, Authorization to access objects, Secure management and administration, and Auditing of behaviours of users and objects.

This paper extends the secure distributed object system model of Varadharajan (1995). The current work presents these extensions and details of the model within the framework of CORBA, covering the security service provided by within the model and identifying the components of the model that provide the services. The discussion proceeds along the security requirements of OMG (1994) as given above. The concept of the Security Facility first mentioned by Varadharajan (1992) is further elaborated, together with the components and functions within the Security Facility. The extended model aims to satisfy the security requirements within CORBA, and possibly within distributed object systems in general.

In the following section we will briefly discuss the OMA/CORBA standard which will be used in the remainder of the paper as the framework for discussion on our extended and more detailed model. The extended model is presented in Section 3, which consist of a number of subsections, including the assumptions underlying the model. Section 4 closes the paper with some comments and remarks.

\section{OMA/CORBA}

The Common Object Request Broker Architecture (CORBA) represents an open standard of application interoperability within the OMA put forward by the OMG. It is, therefore, useful to briefly review the relevant parts of CORBA. 


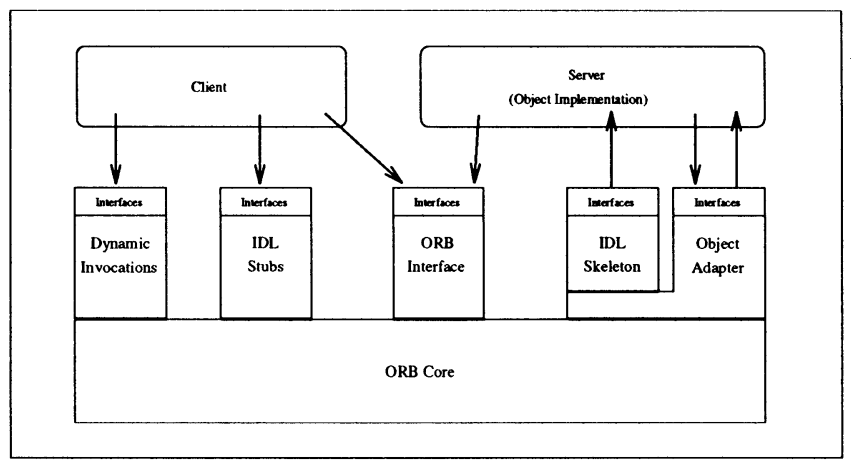

Figure 1 The CORBA Generic

Interfaces

\subsection{CORBA}

The OMG's approach to distributed objects is realized in the Common Object Request Broker Architecture (CORBA). The main service of CORBA is to deliver requests and responses between a "Client" and the Object Implementation or "Server". Here, given the nature of objects within distributed systems, a Client can become a Server, and vice-versa.

This service of delivering requests and returning responses can be achieved by CORBAcompliant systems using two approaches: dynamically or statically. In both approaches, the interfaces are expressed in the Interface Definition Language (IDL) which defines the types of objects based on their allowable operations and the parameters corresponding to these operations. The IDL represents a uniform specification language used to define the interfaces, independent of the execution language. An Interface Repository (IR) may be used to store collections of interfaces of Client and Server objects available in the distributed object system (OMG, 1991).

CORBA has a number of interfaces which allows a Client to direct a request to a given Server (or Object Implementation) without the Client needing to know the actual location and implementation aspects of the Server.

The interfaces in CORBA are shown in Figure 1. Here, when a Client issues a request using either the Dynamic Invocation interface or via an IDL stub, the Server (Object Implementation) receives the request in the form of an up-call via the IDL skeleton. It is the task of the Object Request Broker (ORB) to locate the implementation code and deliver the necessary parameters to the Server.

On its part, the Client must initially obtain the Server's Object Reference (OMG, 1991) which is the information required to specify the Server. The Server may access the services provided by the ORB, and it does so via the Object Adapter. 


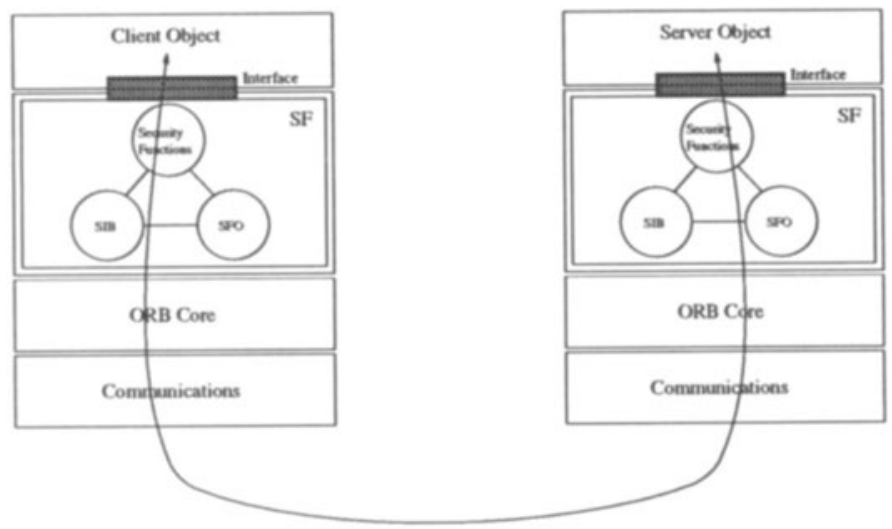

Figure 2 Client and Server Interactions via the Security Facility

\section{A MODEL FOR DISTRIBUTED OBJECT SECURITY}

In the work of Varadharajan (1995) a model for distributed object systems security was presented in terms of a number of security-related services that should be available to objects. In this section we briefly describe the model and extend it to include other aspects of object security.

We will assume that there are in general two types of objects, namely machine (host) objects (which we will refer to as machines), and application and user objects (which we will refer to as just objects). In the model of Varadharajan (1995) the security services directly relating to machines and objects are captured within the Security Facility (SF) component of the system. In terms of the Object Management Architecture (OMA) SF can been viewed as an extension of the ORB Core*. This implies that for secure interactions between the Client and the Server via the ORB, both the Client and the Server must rest on this security-extended ORB. Finally, this Security Facility in the extended ORB must be within the Trusted Computing Base (TCB) boundary. Figure 2 depicts this relationship.

\subsection{Components of the Security Facility}

The Security Facility contains a number of components relevant to carrying-out securityrelated operations on a machine-to-machine level and on an object-to-object level. Each machine within the distributed object system carries a full implementation of the Security Facility. In addition, an external and independent trusted third party is also employed for authentication purposes, namely the Security Server (SS) (which can be an Authentication

${ }^{*}$ It results in a secure ORB. 
Server (AS) and/or a Certification Server (CS)). The Security Server also contains a full implementation of the Security Facility.

The Security Facility is responsible for examing each request for the objects under its protection and decide whether to grant the request based on the authentication and authorization information. The totality of authentication and authorization information available for making the decision can come from (a) the target object and target Security Facility (b) from the Security Server, and (c) from its own local Security Facility.

The main components of the Security facility are:

\section{Security Functions}

The Security Functions contains the mechanisms that are required for the provision of security services. These include cryptographic functionalities such as encryption/decryption, signatures, hashing, and random number generation. For instance, Security Functions will be used in the confidentiality and integrity protection of messages from the source object to target object.

\section{Security Information Base}

The Security Information Base (SIB) contains security-related information and parameters pertaining to users and objects. This is a distributed entity. Each machine has a Local SIB (LSIB) which contains relevant information about its objects. When referring to inter-machine dialogue between objects, two types of SIBs are involved, one being the LSIB and the other the Remote SIB (RSIB).

In addition, we have a SIB in the Security Server, which is referred to as the Security Management SIB (SMSIB). The SMSIB contain security information and parameters pertaining to the machine and user objects in a distributed environment. Typically the environment will be a domain or a realm having a collection of machines, users and the associated objects. Each object has unique universal identifier (UID) which is assigned by the system at the time of its creation. The SIB maintains information relating to the UID and associated security parameters such as the keys used in an interaction, and the types and characteristics of the security services and mechanisms to be used in the object interactions. In a user interaction involving client and server objects, the client object is treated as acting on behalf of the user. Following the authentication process, the user and the object UIDs are used by the access control service.

\section{Secure Factory Objects}

The Secure Factory Objects (SFO) are the collection of objects which are involved in the creation and deletion of other objects. SFOs reside within the Security Facility (SF). Any request from an object (local or remote) to create a new object must be carried-out by an SFO within the corresponding SF. It is important to ensure the integrity and the correct execution of the SFOs.

\section{Secure Interfaces}

Each component within the Security Facility has an interface through which the other components interact. The Security Functions component has one interface with the object in the higher layer, and another interface with the lower communications sublayer. Any interaction between objects within a machine goes through the Security Functions component of the Security Facility. Similarly, any interaction to/from objects in an external machine use the Security Functions component. There are also interfaces between Security Functions and SIB, Security Functions and SFO, and SIB and SFO. 


\subsection{Assumptions}

Let us now consider the assumptions relating to CORBA that need to be taken into account when specifying the security model.

Generic Assumptions

Transparency of Security Services. Security services should be made as transparent as possible to the users while at the same time ensuring that they cannot be bypassed. This implies that the Security Facility functionalities must be implemented as part of the TCB.

At a different level, a certain level of transparency can also be provided at the object level. For instance, a client object need not be necessarily aware of the details of the security mechanisms employed by the Security Facility when the object invokes operations on another (server) object.

Minimal impact on CORBA. We assume that the Security Facility will have minimal impact on the functionality of CORBA and on the performance of the distributed object system. This assumption is necessary for the security model to be practical. In particular, the use of computationally intensive security mechanisms such as the use of public key schemes should be done only where it is necessary.

Scalability. The security model should be able to cope with small as well as large scale distributed systems both intra and inter-enterprise.

Interoperability. The model should allow interoperability between objects using security services and those which do not.

Trust Assumptions

The Security Facility cannot be by-passed. We will assume that the SF is correctly developed, and its functions are properly enforced. The Security Facility basically performs a role similar to that of a Reference Monitor, as it provides security controls for all interobject interactions.

Trust level of Security Facilities. We will assume that all implementations of the Security Facility are trusted to the same level, and that the SFs trust each other to operate correctly.

Security Server Trust. We will assume that the Security Server operates correctly, and the information stored at the SMIB are secure.

\subsection{Authentication}

In terms of the model of Varadharajan (1995), authentication service should allow for user, machine (SF) and object authentication.

The philiosophy behind our authentication service design is as follows: User is authenticated by the Security Facility of the local machine in which the user logs on in cooperation with the Security Server in the domain. When the user invokes an object in a machine, it is the local Security Facility which determines the authentication of the user-object link indicating that the particular object is now acting on behalf of the specific user. At the end of the authentication process, key(s) that can be used for source and target objects interaction is established. In general, the combination of smartcards and authentication mechanisms via a trusted party (based on a strong cryptographic protocol) can provide for user identification, object authentication and for secure channel establishment. We describe below how these are achieved.

Each machine within a domain has a public key, a certified copy of which is obtainable 
by any entity from the Security Server. The secret key corresponding to the public key is stored securely within the LSIB of the Security Facility of the given machine. The LSIB also holds the public key of the Security Server which must be held in a tamper-proof manner, even though the key is publicly known. The SIB may also hold a list of other machine names together with their public keys, with the Security Server holding the most up-to-date version of the [machine, public-key] pairs.

First consider a user $U$ logging into a machine $M$. We describe one scheme using challenge-response technique. We will assume that the user and machine have agreed on a publicly available one-way function. We also assume that the user has registered his security information (key/password) with the Security Server. Upon login, a challenge is issued either by the local SF or by the Security Server through SF to the user. The user calculates the response by applying the publicly known function and his secret information (key/password) to the challenge. The response is returned to the Security Server which verifies it and confirms the user identity to the SF. If a public key approach is used, then the calculation of the response includes signature using the user's private key. If a symmetric key approach is used, then the response calculation involves the use of the secret key initially registered at the Security Server.

Now consider machine (SF) to machine (SF) authentication. Recall that the public keys of the SFs are available from the Security Server in the form of certificates. Hence when SF1 wishes to authenticate itself to SF2, SF2 may send a challenge and SF1 can calculate the signed response using its private key and return it to SF2. SF2 can obtain SF1's certificate from the Security Server and verify the response from SF1. At the end of the authentication process, a dialogue key between a pair of object interactions can be established. For instance, a symmetric key to be used to protect communications between objects $\mathrm{O} 1$ in machine M1 and $\mathrm{O} 2$ in machine M2. Once established, the dialogue keys are stored in the LSIB of the SF. The dialogue keys have associated with them a timeout period. In general, the level of granularity of these dialogue keys can be either on an objectto-object basis or an object-group-to-object-group basis. Note that the authentication service uses public key technology whereas symmetric key technology is used to provide confidentiality and integrity of inter-object communications.

Now consider a scenario where a user $\mathrm{U}$ employs an object $\mathrm{O} 1$ residing on machine $\mathrm{M} 1$ with SF1 to interact with object O2 residing on machine M2 with SF2.

* Authentication of user U. As mentioned above, done by SF1 in cooperation with the Security Server.

* Authentication of SF1. Done by SF2. The transferred authentication information from SF1 to SF2 includes the user and object UIDs signed by SF1 using public key technology. For mutual authentication, a similar procedure with SF1 verifying SF2's signed information. If needed, the required certificates are obtained from the Security Server.

* Authentication of object O1. First verified by SF1 when it receives a request from O1 acting on behalf of U. At the receiving end, SF2 verifies O1's authenticity based on SF1's signature. $\mathrm{O} 2$ accepts O1's authenticity based on SF2's verification.

In general, there are two approaches when it comes to whether the source (client) object or the target (server) object should provide or fetch the necessary authentication information to do the verification. Our approach has been to make the client gather the necessary information (e.g. from the Security Server) and provide it to the target for verification - the push approach. Where the nature of the security information is dynamic, 
it is necessary for the target (server) object to get hold of the uptodate information (e.g. revoked certificates).

\section{Authentication Aspects in Creation and Deletion of Objects}

Within the context of the current extended model the creation and deletion of objects are performed by the Security Factory Objects (SFO) located within the Security Facility. When a new object is created by the SFO a unique UID and security parameters for the new object's interactions are specified by the SFO. The latter includes whether the new object requires confidentiality and/or authentication services, integrity services, characteristics of algorithms and types of keys used. The association between the new object, the UID and security information associated with the object is stored in the Local SIB of the machine where the new object resides. If the object has domain-wide relevance, then the information about the object are passed onto the SMIB at the Security Server. Note that the LSIB also has security parameters which emanate from the Security Server, which is the result from the domain-wide security policies.

An important issue related to the creation of objects is the location of the code for the new object. In the current model, the code for such new objects resides at the machine on which the generating SFO also resides.

When an object (remote or local) requests the creation/deletion of an object (or other operations), the authorization of the request must be validated. Here, two important points need to be noted. First, when the client object wishing to create a new object resides within the machine where SFO resides, the path from this local object to SFO is established without the aid of cryptographic techniques. Any such request to SFO must be via the Security Functions component of the local machine. Second, when the client object wishing to create a new object using a SFO of a remote machine, the path between the requesting object and the SFP needs to be secure. As mentioned above, this is done by providing an authenticated and secure channel using cryptographic techniques. This path consists of three segments, namely the segment between the requesting object and the SF at the local machine, the segment between the local and remote machines, and finally the segment between the communication sublayer and the SFO in the remote machine.

\subsection{Access Control}

The main role of the access control service is to apply control over messages between objects. In providing the access control service, we need to decide the following :

* What access control information is used in the decision making process?

* What type of access control rules and policies can be supported?

* What access control authorities are needed

Returning to the scenario, we have an object $\mathrm{O} 1$ in a machine M1 with SF1 wishing to make a request on another object $\mathrm{O} 2$ on a machine M2 with SF2.

Access Control Information and Rules

The access control information used in the specification of access rules can be based around some attributes of the source object $\mathrm{O} 1$, the target object $\mathrm{O} 2$, and the action being requested.

Typical attributes of the source (initiator) and the target objects that are used in the rules are: the identity of the object, the class of the object, the identity of the owner of the object, the membership of the owner or object in some domain, his or her role, privileges 
associated with the objects, and sensitivity/integrity labels associated with the objects. Hence subjects of access control are objects, users and groups of objects and users. As for the action being attempted, two approaches are possible. First, the decision may be based simply on the message type. Secondly, the decision may be based on more fundamental properties: the familiar "know-about", "read", "modify", "move" and "delete", and so on.

As an example, the model allows rules such as Object $O 1$ in Class C1, owned by User X, can invoke Method $Y$ on Object O2. More generally, it allows specification of policies that have constraints at various levels of granularity: machine, object, method and method and parameters based. Hence one can specify identity-based, role based, label based and capability based access control policy rules.

The model should be able to handle both positive and negative authorizations. In this paper, we will not go into details of the overriding semantics in the access control rules and the conflict resolution procedures.

\section{Access Control Authorities}

The access mediation process consists of two parts: First the access request by an object for an action on another object needs to evaluated using the relevant attributes of the objects in question and the specified access control rules. We will refer to the entity which performs this function as Access Decision Facility (ADF). Secondly, the decision is enforced thereby either allowing the access request or denying it; this is carried out by an Access Enforcing Facility (AEF). Now the question to consider is how many such $\mathrm{AEF}$ and ADFs do we have for our application and how are they distributed? Several alternatives are possible, as we describe below.

First, note that as there is no notion of binding between objects, it seems necessary that access control checks need to be performed call by call. Secondly, we assume that in our model there is no central access control authority which decides who can access what servers. Thus there are three possible locations for authority: the initiator SF, the target $\mathrm{SF}$, and the target object implementation itself.

The initiator SF can enforce control over outgoing messages so that an initiator may not perform an action which is not in conformance with the policy for the initiator. The target SF can enforce control over the incoming messages so that the target object may not receive a request that is not in conformance with the access control policy for the target. The target object itself may perform additional controls to decide whether to grant the request or not.

Enforcing control at the initiator will help to eliminate some calls at the local end, thereby increasing efficiency. Enforcement at the target may be adequate for many situations whereas there may be certain circumstances where at least some of the enforcement needs to be performed by the destination object implementation itself. Note that there is a trust issue here with respect to leaving the access decision and enforcement at the object itself.

There is also the case when understanding of the semantics of the message is required in making the access decision. For instance, a given message may result in processing which creates, deletes or moves objects, under an arbitrary message type. If the access policy at the SF is described in terms of read, modify, destroy and so on, the SF will not be able to interpret the message in these terms. One possibility may be for the SF to pass on access information to objects in the form of hints, expressed in terms of read, modify, 
destroy and so on, and let the object to make the decision based on these. However, this will place extra trust assumptions on the object.

Alternatively, access control rules can be defined in terms of the message types. Creation of objects requires their entry in the access control scheme. This can be taken care of using the primitives provided by the SF. Given that the SF is always involved in object creation, the involvement of the local SF in access control decision can help solve the problem of the correspondence between message types and object creation.

Related to the decision of $\mathrm{ADF}$ and $\mathrm{AEF}$ placement is the problem of the location of information representing the state of access control. It may make sense to associate information on restrictions on access to an object with the object itself. However it is appropriate to store such information in the SF which may be protected and trusted, whereas an object may not be.

Feedback to callers needs to be designed. If a user tries to access an object on the remote machine, five things may happen: the object might not exist; the object might exist but fall outside the restricted view granted to the user by the owner of the owner of the remote machine; the object may not support the chosen message type; the object may not be allowed to respond the chosen message type under the restricted view; the object may be allowed to respond to a supported message type. It is desirable that the responses to the remote user in the first and second cases, and in the third and fourth cases, be the same. For the third case, this may require some discipline on the part of the developer.

\subsection{Delegation}

An important requirement that commonly arises in cooperating object-oriented systems that is associated with access control is that of delegation. The principle behind delegation is that one object can authorize another object "to act on its behalf". Delegation allows a more flexible form of access control.

Returning to our scenario, the delegation situation occurs when the object $\mathrm{O} 1$ authorizes an object $\mathrm{O} 2$ to make a request on his behalf to object $\mathrm{O} 3$. Secure delegation is achieved by verifying that an object that claims to be acting on another's behalf, is indeed authorized to act on its behalf. The problem of delegation in distributed systems has been addressed in detail by Varadharajan et al. (1991). In this model, we use the nested or linked delegation schemes proposed by Varadharajan et al. (1991).

Let us consider two cases of delegation:

Case D1: Objects $\mathrm{O} 1$ and $\mathrm{O} 2$ reside on machine $\mathrm{M} 1$, while object $\mathrm{O} 3$ resides on machine M3.

Case D2 Object $\mathrm{O} 1$ resides on machine $\mathrm{M} 1$, object $\mathrm{O} 2$ resides on machine $\mathrm{M} 2$, and object $\mathrm{O} 3$ resides on machine $\mathrm{M} 3$.

From the point of view of $\mathrm{O} 3$, the following must be validated:

* Whether $\mathrm{O} 1$ has the privileges to perform the requested operations in O3.

* Whether $\mathrm{O} 1$ has delegated its privileges to $\mathrm{O} 2$.

* Whether it is $\mathrm{O} 2$ that is truly making the request to $\mathrm{O} 3$.

In Case D1, where $\mathrm{O} 1$ and $\mathrm{O} 2$ reside on machine M1 the issue of whether $\mathrm{O} 1$ has the privilege to delegate to $\mathrm{O} 2$ must be resolved by SF1 on M1 in the usual manner. The issue of whether $\mathrm{O} 1$ has delegated $\mathrm{O} 2$ must also be left to SF1. The machine SF3 must trust the signature from SF1 in M1 to verify that SF1 has validated the O1-to-O2 delegation. 
Finally, the problem of determining whether it is $\mathrm{O} 2$ that is making the request to $\mathrm{O} 3$ is resolved by the verification of SF1's signature on O2's request and the trust on SF1 to have correctly authenticated $\mathrm{O} 2$.

In Case D2 the target object O3 must receive a token signed by SF1 in M1 stating that $\mathrm{O} 1$ possesses the right to perform delegations. In addition, the token must also indicate that it is $\mathrm{O} 2$ that has specifically received the delegation from $\mathrm{O} 1$ in this instance. The issue of verifying that it is $\mathrm{O} 2$ that is making the request is achieved using the usual object to machine (O2 to SF2) authentication procedures along with SF2's signature on the request.

\section{CONCLUDING REMARKS}

In this paper we have discussed the security issues relating to distributed object systems and presented an extended model of the secure distributed object model of Varadharajan (1995). We considered the incorporation of the model into the CORBA framework, by extending the ORB with a Security Facility.

The role and functions of the Security Facility have been described in detail. We described the main components of the Security Facility namely the Security Functions component, the Security Information Base component, the Secure Factory Objects component. The location of the Security Facility with respect to the objects in the system, the ORB, and the communications sublayer has been identified. The assumptions made by the security model have also been outlined.

The provision of security services for object interactions have been described. Services included user and object authentication, secure creation and deletion of objects by Secure Factory Objects, access control between objects, and delegation between objects residing on different machines.

The intention of this paper was not to describe all the details of the model but to highlight the design choices that need to carefully considered when developing a security model for distributed object systems. The issues discussed in the design of object security services are applicable to distributed object-oriented applications in general ${ }^{\dagger}$, and in particular to implementations of distributed object systems conforming to the CORBA standard.

\section{REFERENCES}

Fernandez, E. B., Gudes, E., and Song, H. (1989) A security model for object-oriented databases. In Proceedings of the 1989 IEEE Symposium on Security and Privacy (Oakland, CA, 1989), IEEE Computer Society, pp. 110-115.

Keefe, T. F., Tsai, W. T., and Thuraisingham, M. B. (1989) SODA: A secure objectoriented database system. Computers \& Security 8, 6 (1989), 517-533.

Kim, W. (1990) Object-oriented databases: Definition and research direction. IEEE Transaction on Knowledge and Data Engineering 2, 3 (September 1990), 327-341.

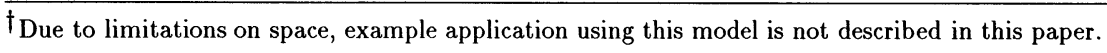


Lunt, T. F. (1989) Multilevel security for object-oriented database systems. In Database Security III: Status and Prospects (Results of the IFIP WG 11.3 Workshop on Database Security) (Monterey, CA, 5-7 September 1989), D. L. Spooner and C. Landwehr, Eds., IFIP, North-Holland, pp. 199-209.

Object Management Group (1991) The Common Object Request Broker: Architecture and Specification. OMG Document Number 91.12.1.

Object Management Group (1994) White Paper on Security. OMG Document Number 94.4.16.

Thuraisingham, M. B. (1989) A multilevel secure object-oriented data model. In Proceedings of the 12th National Computer Security Conference (Baltimore, MD, October 1989), NIST/NCSC, pp. 579-590.

Varadharajan, V. (1992) A security reference model for a distributed object system and its applications. In Proceedings of the 15th National Computer Security Conference (Baltimore, MD, 1992), pp. 593-619.

Varadharajan, V. (1995) Distributed object systems security. In Information Security - the Next Decade: Proceedings of the 11th International Conference on Information Security IFIP/SEC'95 (Johannesburg, 1995), J. H. P. Eloff and S. H. von Solms, Eds., Chapman \& Hall, pp. 305-321.

Varadharajan, V., Allen, P., and Black, S. (1991) An analysis of the proxy problem in distributed systems. In Proceedings of the 1991 IEEE Symposium on Research in Security and Privacy (Oakland, CA, 1991), IEEE Computer Society, pp. 255-275.

Varadharajan, V., and Black, S. (1991) Multilevel security in a distributed object-oriented system. Computers \& Security 10, 1 (1991), 51-68.

\section{Vijay Varadharjan}

Vijay Varadharjan has taken up the Professor and Chair of Computing Science at the University of Western Sydney, Australia, from Jan.1995. Prior to taking up this appointment, he was heading the Distributed Systems and Networks Security Research Group at Hewlett-Packard Research Labs., European Headquarters, U.K. since 1989. Prior to HP, he was a Research Manager at British Telecom Research Labs. U.K., from 1987 till 1988. From 1985 till 1987, he was Research Fellow and Lecturer in Computer Science at Plymouth and Reading Universities. Vijay did his Ph.D in Computer and Communication Security in the U.K in 1984.

He has managed and carried out numerous projects in secure systems for Hewlett-Packard and British Telecom. He has done several consultancies for organizations in the US and the UK. He has also worked on European Commission's Projects related to Security. He has published and presented over 60 papers in International Journals, Conferences, and Industrial Fora in US and Europe. He has also participated in several International Standards activities (ISO, OSF, ECMA, IEEE). His technical interests are Distributed System Security, Networks Security, Security Architectures, Protocols, Formal Models and Evaluation.

Vijay is a Fellow of the British Computer Society, a Fellow of the IEE, a Fellow of the IMA.

\section{Thomas Hardjono:}

Thomas Hardjono obtained the B.Sc. (Hons) degree in Computer Science from The University of Sydney and the Ph.D. degree also in Computer Science from The University of New South Wales, Australia, in 1987 and 1991 respectively. From 1992 to 1993 he was a Research Scientist at the ATR Communication Systems Research Laboratories, in Kyoto, Japan. Currently he is a Research Fellow at the Centre for Computer Security Research at the University of Wollongong, Australia. He also lectures at the University of Western Sydney - Macarthur. His research interests include security in network and ditributed systems, database systems and cryptography. Thomas Hardjono is a member of the ACM, IEEE and IEICE. 